\title{
Distribuição intra-planta e flutuação populacional de Tegolophus brunneus (Acari: Eriophyidae: Phyllocoptinae) em tangerineira
}

\author{
Intra plant distribution and populational fluctuation of Tegolophus brunneus Flechtmann, \\ 1999 (Acari:Eriophyidae: Phyllocoptinae) in mandarin tree. \\ Luciana Ribeiro Bressan \\ Ana Paula Ott \\ Universidade Federal do Rio Grande do Sul- UFRGS - Porto Alegre - Rio Grande do Sul - Brasil
}

\section{Resumo}

Este estudo teve como objetivo conhecer a distribuição intra-planta e a sazonalidade de Tegolophus brunneus Flechtmann, 1999 (ERIOPHYIDAE) em folhas de tangerineiras 'Murcot' (Citrus sinensis L. Osbeck $x$ Citrus reticulata Blanco) produzidas sob manejo orgânico em Montenegro, RS no período de maio de 2012 a fevereiro de 2013. O estudo foi realizado a partir do exame de folhas jovens e maduras, retiradas da parte interna e externa da copa no terço médio de cada um dos quadrantes de plantas escolhidas aleatoriamente. Todas as folhas amostradas tiveram sua face adaxial e abaxial avaliada quanto à presença de ácaros. No total foram registrados 6.641 indivíduos de $T$. brunneus. A análise de sua distribuição indica que este ácaro localiza-se preferencialmente na face adaxial de folhas maduras presentes na região externa da copa localizadas no quadrante norte das tangerineiras. A maior população ocorreu em fevereiro, não havendo registro de indivíduos de agosto a outubro.

\section{Abstract}

This study aimed to assess the intraplant distribution and seasonality of brown citrus rust mite Tegolophus brunneus Flechtmann, 1999 (Acari: Eriophyidae), in 'Murcot' mandarin leaves (Citrus sinensis L. Osbeck x Citrus reticulata Blanco) produced under organic management. Samples were accomplished montly in Montenegro, RS, from May 2012 to February 2013. The study was accomplished through the examination of young and mature leaves, taken from the inner and outer portions of the middle third of the canopy in each quadrants of randomly choosen plants. All sampled leaves had their adaxial and abaxial faces evaluated regarding the presence of mites. In total, 6,641 individuals of $T$. brunneus were registered. The distribution analysis indicates that this mite is preferentially located on the adaxial side of mature leaves present in the outer region of the canopy located in the northern quadrant of the mandarins. The largest population occurred in February, and there were no records of individuals from August to October

\section{Palavras-chave}

Acari. Ácaro-marrom-daferrugem-dos-citros. Distribuição espacial. Sazonalidade. Cultivo orgânico.

\section{Keywords}

Acari. Brown citrus rust mite. Spatial distribution. Seasonality. Organic management. 


\section{Introdução}

Tegolophus brunneus Flechtmann, 1999 foi descrito a partir de amostras de folhas e frutos de laranjeiras e tangerineiras com sintomas de ferrugem, principalmente em frutos, provenientes de pomares do Estado de São Paulo. Até então, o único ácaro da ferrugem registrado no Brasil era Phyllocoptruta oleivora (Ashmead, 1879). Morfologicamente, $T$. brunneus é muito similar à Tegolophus australis Keifer, 1964 de ocorrência australiana, ambos apresentam empódio com 4 raios (FLECHTMANN, 1999). T. brunneus apresenta o primeiro anulo dorsal similar aos ânulos subsequentes, enquanto o primeiro tergito em $T$. australis é largo e áspero; as fêmeas de T. brunneus apresentam epigínio com 12 a 14 elevações longitudinais, enquanto em T. australis são 18; elevação dorso central estendendo-se por todo comprimento do tanosoma em $T$. brunneus, enquanto em $T$. australis esta inicia no $3^{\circ}$ tergito e esvanece no $16^{\circ}$ tergito (FLECHTMANN, 1999).

Tegolophus brunneus tem sido registrado em pomares cítricos de diferentes regiões brasileiras. Albuquerque (2006) considerou a espécie como abundante e constante em pomares cítricos com cultivo orgânico em Jaguariúna, São Paulo. Moraes e Flechtmann (2008) comentam o registro desta espécie em mudas cítricas no estado do Acre, oriundas do estado de São Paulo. Bobot et al. (2011), em levantamento da acarofauna em Citrus sinensis L. Osbeck no estado do Amazonas, registraram $T$. brunneus como a segunda espécie mais abundante nas folhas cítricas apresentando alta variação na abundância ao longo do ano. No estado do Rio Grande do Sul, foi coletado em pomar de laranja Valência com manejo convencional, porém sem tratamento fitossanitário durante as amostragens no município de Taquari, sendo considerada de ocorrência constante ao longo de um ano de amostragem (HORN et al. 2011). Estes estudos apontam uma ampla distribuição da espécie no País com presença constante e alta abundância nas áreas citrícolas.

Sobre a bioecologia de T. brunneus, sabe-se que inclui indivíduos errantes que foram coletados em folhas de frutos da laranja e tangerina e que provocam ferrugem nas folhas, principalmente nos frutos (FLECHTMANN, 1999). Dados sobre a distribuição espacial e flutuação populacional de eriofídeos em citros (OLIVEIRA, 2007; TORRES, 2012; SILVA et al., 2012) têm permitido que se conheça mais sobre a bioecologia das espécies, contribuindo para o desenvolvimento de planos de amostragem confiáveis para o estabelecimento do nível de controle, indispensável no manejo integrado de ácaros de importância agrícola.

O objetivo deste trabalho foi conhecer a distribuição intra-planta e a flutuação populacional de $T$. brunneus em folhas de tangerineiras, além de conhecer sua sazonalidade.

\section{Material e métodos}

O trabalho foi realizado no período de maio de 2012 a fevereiro de 2013, no município de Montenegro, localizado no Vale do Caí, Estado do Rio Grande do Sul (2968'32" S e $51^{\circ} 46^{\prime} 07^{\prime \prime} \mathrm{O}$ ), em dois pomares de tangerineiras tangor 'Murcott' (Citrus sinensis L. Osbeck $\mathrm{x}$ Citrus reticulata Blanco) enxertados sobre Poncirus trifoliata (L.) de aproximadamente 21 anos de idade, com área de 1 ha, apresentando espaçamento de $3,5 \mathrm{~m}$ entre plantas e de $5 \mathrm{~m}$ entre linhas.

Os pomares são manejados de acordo com o sistema de cultivo orgânico desde sua implantação e para sua manutenção aplica-se anualmente chorume (líquido originado da 
decomposição da matéria orgânica) e a cada dois anos é aplicado composto orgânico oriundo de resíduos industriais. Utilizam-se como medidas fitossanitárias de controle de pragas, a aplicação de calda bordalesa três vezes ao ano e anualmente calda sulfocálcica. É realizada roçada nas entrelinhas uma vez por ano, antes da colheita dos frutos.

Mensalmente, de cada pomar, foram avaliadas sete plantas escolhidas aleatoriamente de onde foram retiradas as folhas do terço médio das tangerineiras. Não houve repetição de plantas ao longo do experimento. Para análise da distribuição de $T$. brunneus nas tangerineiras, dividiu-se cada planta em quadrantes (norte, sul, leste e oeste) e de cada quadrante foram retiradas duas folhas para análise da idade das folhas (1 madura e 1 jovem) e duas folhas para análise da posição na copa (1 copa interna e 1 copa externa), além de oito folhas adicionais (duas de cada quadrante). A cada amostragem foram coletadas 24 folhas/planta, totalizando 168 folhas/pomar. Todas as folhas retiradas foram utilizadas para análise da presença de $T$. brunneus nos quadrantes, na face das folhas (adaxial e abaxial) e para análise da sazonalidade.

As folhas foram armazenadas em sacos plásticos hermeticamente fechados, etiquetados, acondicionados em caixa térmica e transportadas ao laboratório. Todos os exemplares de $T$. brunneus observados sob microscópio estereoscópio foram retirados com pincel de ponta fina $\left(n^{\circ} 00\right)$ e montados em lâminas de microscopia em meio de Hoyer. Estas permaneceram de sete a dez dias em estufa com temperatura entre 45 a $55^{\circ} \mathrm{C}$ para fixação, distensão e clarificação dos espécimes (JEPPSON et al., 1975). A determinação taxonômica foi baseada na descrição da espécie (Flechtmann, 1999) e realizada em microscópio biológico com contraste de fase pelo autor sênior. Os espécimes foram agregados à coleção didática de referência do Laboratório de Acarologia Agrícola da UFRGS, Departamento de Fitossanidade, Faculdade de Agronomia, Porto Alegre, RS e Vouchers foram depositados no MCN-FZBRS (R. Ott).

Para detectar diferenças significativas em relação à presença de $T$. brunneus nos quatro quadrantes foi realizada análise multivariada através do teste de Tukey $(P<0,05)$. A preferência de $T$. brunneus em relação à idade e face da folha e posição na copa foi testada com o Teste Mann-Whitney $(P<0,05)$. A análise estatística foi processada através do programa SigmaStat Versão 3.5 (DUNDAS SOFTWARE LTDA, 2006). Foi utilizada a Correlação de Spearman (Past versão 1.79) para identificar associações entre a sazonalidade de $T$. brunneus e temperatura máxima, temperatura mínima e precipitação.

\section{Resultados e discussões}

As maiores populações de $T$. brunneus nas tangerineiras analisadas foi registrada no quadrante norte $(H=17,330, P=<0,01)$ (Tab. 1). Este resultado corrobora estudos realizados com plantas cítricas no Hemisfério Norte (HALL et al. 1991; WALKER et al. 1992; LINDQUIST et al. 1996), que indicam a maior presença de eriofídeos em quadrantes com maior incidência de luminosidade.

A maior população de $T$. brunneus ocorreu nas folhas maduras das tangerineiras $(t=2,935 ; P=<0,001)$ (Tab. 1). Gouvea et al.(2006) relatam que folhas mais velhas de erva-mate são mais favoráveis ao desenvolvimento de Dichopelmus notus Keifer, 1959 (Eriophyidae). Esta preferência pode estar relacionada às características nutricionais dos diferentes estágios 
fenológicos da planta, o que já foi constatado por Potter e Kimmerer (1989) e pode estar ocorrendo com T. brunneus em citros.

A maior população de $T$. brunneus foi registrada na face adaxial das folhas de tangerineiras $(U=11885,000 ; P=<0,001)$ (Tab. 1). Manson (1972) registrou $T$. australis ocorrendo na face adaxial de Citrus sp., corroborando o registrado no atual estudo. Calacarus heveae Feres, 1992, espécie também pertencente à Phyllocoptinae foi registrada ocorrendo em maior abundância na face adaxial de folhas de seringueiras (De Vis et al. 2006; Ferla et al. 2007).

Em relação à copa das tangerineiras, $T$. brunneus apresentou maior número médio na copa externa ( $U=3063,00 ; P=0,009)$ (Tab. 1). Landeros et al. (2003) também registraram a maior ocorrência de $P$. oleivora em laranja Valencia no México, na parte externa das plantas cítricas.

Os resultados relativos à maior abundância de $T$. brunneus quanto à face da folha, à copa da planta e ao quadrante, podem ser interpretados como preferências intrínsecas da espécie às condições abióticas. A análise da distribuição intra-planta de $T$. brunneus indica que este ácaro localiza-se preferencialmente na facial adaxial de folhas maduras presentes na região externa da copa localizadas no quadrante norte das tangerineiras.

A flutuação populacional de $T$. brunneus (Fig. 1), apresentou menor número de maio a julho/2012 e de agosto a outubro/2012 nenhum indivíduo foi registrado. A partir de novembro/2012 o número médio de indivíduos aumentou, alcançando as maiores populações em janeiro e fevereiro/2013, sendo esta mais de 50 vezes maior do que as médias registradas nos meses anteriores. Bobot et al. (2011) registraram a flutuação sazonal de $T$. brunneus e $P$. oleivora, aumentando mais de 50 vezes em fevereiro em relação aos demais meses, sugerindo que as duas espécies são fortemente afetadas por alta temperatura e alta pluviosidade. Este padrão de poucos indivíduos nos meses de inverno e início de primavera é confirmado por Horn et al. (2011), que coletaram a maior população em fevereiro e não registraram nenhum espécime de $T$. brunneus de junho a outubro também no estado do Rio Grande do Sul.

Em relação à influência de fatores abióticos como temperatura e precipitação sobre a sazonalidade de $T$. brunneus, a correlação de Spearman constatou associações positivas entre a sazonalidade da espécie e as temperaturas máximas ( $\mathrm{rs}=0,870, \mathrm{P}=<0,050$ ) e mínimas $(\mathrm{rs}=0,854, \mathrm{P}=<0,050)$. Não foi evidenciada correlação entre a abundância de $T$. brunneus e a precipitação ( $r s=0,0246, \mathrm{P}=>0,050$ ).

A influência da temperatura sobre $T$. brunneus registrada no atual estudo é similar ao encontrado por Beattie et al. (1991), para T. australis que também foi altamente influenciada pelo aumento e diminuição da temperatura, apresentando picos populacionais no verão e decréscimo no inverno.

\section{Conclusões}

T. brunneus apresenta maior abundância na face abaxial das folhas maduras da copa externa do quadrante norte das tangerineiras e as maiores populações ocorrem no verão.

\section{Referências}

1. ALBUQUERQUE, F.A. Diversidade de ácaros em cultivo orgânico de citros e na vegetação natural circundante e perspectivas para a criação massal de Iphiseiodes zuluagai (Acari: 
Phytoseiidae). 2006. 108p. Tese (Faculdade de ciências Agrárias e Veterinárias Doutorado) - Universidade Estadual Paulista “Júlio Mesquita Filho", UNESP, 2006.

2. BOBOT, T.E. et al. Mites (Arachnida, Acari) on Citrus sinensis L. Osbeck Orange trees in the State of Amazonas, Northern Brazil. Acta Amazonica, 41, p. 557-566, 2011.

3. DE VIS, R.M.J.; MORAES, G.J.; BELLINI, M.R. Mites (Acari) of rubber trees (Hevea brasiliensis Muell. Arg., Euphorbiaceae) in Piracicaba, State of São Paulo, Brazil. Neotropical Entomology, 35, p. 112-120, 2006.

4. DUNDAS SOFTWARE LTDA. SigmaStat for Windows. Version 3.5. 2006.

5. FERLA, N.J.; MORAES, G.J,; BONATO, O. Distribuição espacial e plano de amostragem de Calacarus heveae (Acari) em seringueira. Iheringia Série Zoologia, 97, p. 447-451, 2007.

6. FLECHTMANN, C.H.W. Tegolophus brunneus n.sp., a new citrus rust mite from Brazil (Acari: Eriophyidae). International Journal of Acarology, 25, p. 265-267, 1999.

7. GOUVEA, A., BOARETTO, L.C., ZANELLA, C.F., ALVES, L.F.A. Dinâmica populacional de ácaros (Acari) em erva-mate (Ilex paraguaiensis St. Hil.:Aquifoliaceae). Neotropical Entomology, Londrina, v.35, n.1, p.101-111, 2006.

8. HALL, D.G.; CHILDERS, C.C.; EGER, J.E. Estimating citrus rust mite (Acari: Eriophyidae) levels on fruit in individual citrus trees. Environmental Entomology, 20, p. 283-390, 1991.

9. HORN, T.B.; JOHANN, L.; FERLA, N.J. Ecological interactions between phytophagous and predaceous mites in citrus agroecossystems in Taquari Valley, Rio Grande do Sul, Brazil. Systematic \& Applied Acarology, 16, p. 133-144, 2011.

10. JEPPSON, L.R.; KEIFER, H.H.; BAKER, E.W. 1975. Mites injurious to economic plants. California, Berkeley: University of California Press, $614 p$.

11. LANDEROS, J. et al. Distribución espacial y fluctuación poblacional de Phyllocoptruta oleivora (Ashmead) AAcari: Eriophyidae) em cítricos de Guemez, Tamaulipas. Acta Zoológica Mexicana, 89, p. 129-138, 2003.

12. LINDQUIST, E.E.; SABELIS, M.W.; BRUIN, J. 1996. Eriophyidae mites: their biology, natural enemies and control. Vol. 6. Amsterdam: Elsevier. 787p.

13. MANSON, D.C.M. New species and new records of Eriophyid mites (Acarina: Eriophyidae) from New Zealand and the Pacific Area. Acarologia, 13, p. 351-360, 1972.

14. MORAES, G.J.; FLECHTMANN, C.H.W. 2008. Manual de Acarologia: acarologia básica e ácaros de plantas cultivadas no Brasil. Holos: Ribeirão Preto. 288p. 
15. OLIVEIRA, W.P. Flutuação e densidade populacional de ácaros (Acari) em três sistemas de produção de citros. 2007. 143p. Tese (Ecologia Aplicada - Doutorado) - Universidade de São Paulo - USP, 2007.

16. POTTER, D.A.; KIMMERER, T.W. Inhibition of herbivory on young holly leaves: evidence for the defensive role of saponins. Oecologia, 78, p. 322-329, 1989.

17. SILVA, M.Z.; SATO, M.E.; OLIVEIRA, C.A.L. Diversidade e dinâmica populacional de ácaros em pomar cítrico. Bragantia, 71, p. 210-218, 2012.

18. TORRES, I.V.R. 2012. Identificación de acaros que afectam cultivos de naranja valencia (Citrus sinensis L.) en el nucleo sur occidental de Colombia y establecimiento de dinamica de poblacion y fenologia de algunas especies de importancia economica. 2012. 198p. Tese. (Ciencias Agropecuarias - Doutorado) - Universidad Nacional de Colombia, 2012.

19. VACANTE, V. 2010. Citrus mites: identification, bionomy and control. Oxfordshire: CABI Head Office. 378p.

20. WALKER, G.P.; VOULGAROPOULOS, A.L.; PHILLIPS, P.A. Distribution of citrus bud mite (Acari: Eriophyidae) within lemon trees. Journal of Economic Entomology, 85, p. 23892398, 1992.

\section{Anexos}

Tabela 1. Número médio de Tegolophus brunneus em folhas de tangerineiras em diferentes quadrantes de maio de 2012 a fevereiro de 2013 em Montenegro, RS, Brasil.

\begin{tabular}{lc}
\hline Quadrante & Número médio ( $\mathbf{n}=\mathbf{3 . 3 6 0 )}$ \\
\hline Norte & $0,34 \mathrm{a}$ \\
Sul & $0,32 \mathrm{~b}$ \\
Leste & $0,31 \mathrm{~b}$ \\
Oeste & $0,18 \mathrm{c}$
\end{tabular}

Números seguidos de letras diferentes na coluna diferem significativamente pelo teste de Tukey a $5 \%$ de probabilidade. 
Tabela 2. Número médio de Tegolophus brunneus em folhas e na copa de tangerineiras de maio de 2012 a fevereiro de 2013 em Montenegro, RS, Brasil.

\begin{tabular}{|c|c|c|c|}
\hline Número médio & $\begin{array}{l}\text { Idade da folha } \\
\qquad(n=1.120)\end{array}$ & $\begin{array}{l}\text { Face da folha } \\
\qquad(n=3.360)\end{array}$ & $\begin{array}{c}\text { Copa } \\
(n=1.120)\end{array}$ \\
\hline \multirow{2}{*}{ T. brunneus } & Madura & Abaxial & Interna \\
\hline & $0,65 b$ & $0,98 a \quad 0,18 b$ & $0,71 a \quad 0,79 b$ \\
\hline
\end{tabular}

Números seguidos de letras diferentes na linha diferem significativamente pelo teste de Tukey a $5 \%$ de probabilidade.

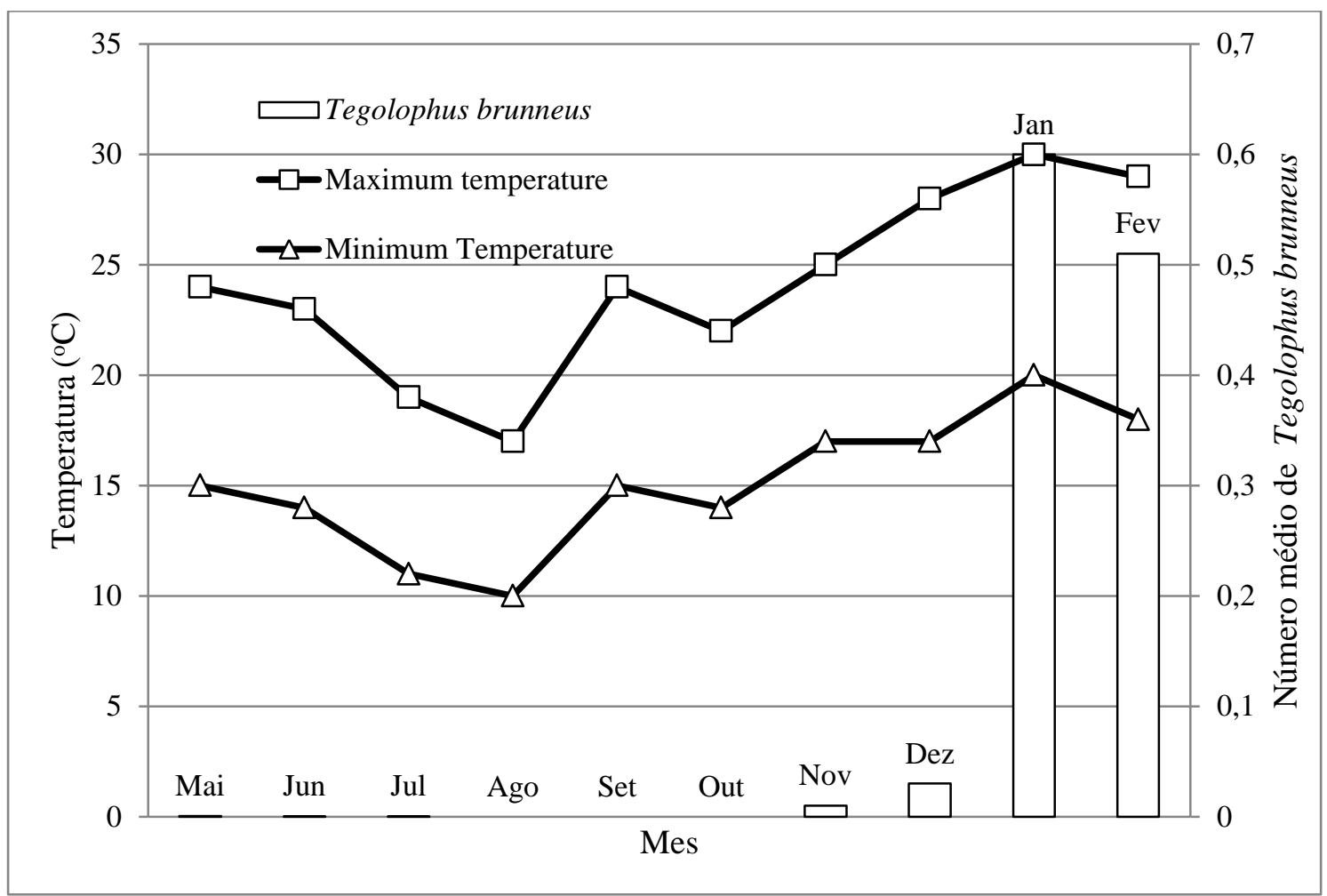

Figura 1. Número médio de Tegolophus brunneus coletados em folhas de tangerineiras de maio de 2012 a fevereiro de 2013 em Montenegro, RS, Brasil. ( $n=3.360$ ). 